\title{
ANALYSIS OF SPONSORED CONTENT IN THE CASE OF COMPETING CONTENT PROVIDERS
}

\author{
Hamid Garmani ${ }^{1}$, Driss Ait Omar ${ }^{2}$, Mohamed El Amrani ${ }^{3}$, Mohamed Baslam ${ }^{4}$ and \\ Mostafa Jourhmane \\ TIAD Laboratory, University Sultan Moulay Slimane, Morocco, \\ 1 garmani.hamid@gmail.com, ${ }^{2}$ aitomard@gmail.com, ${ }^{3}$ med.el.amran@ gmail.com, \\ ${ }^{4}$ baslam.med@gmail.com, 5jourhman@hotmail.com
}

Abstract - With a sponsored content plan in the internet market, the content provider (CP) can pay to the internet service provider (ISP) on behalf of the endusers the network subscription fees. As such, CP, ISP and end-users are incentive to participate in the sponsored content. In this paper, we propose an internet market model with sponsored content. Furthermore, we investigate the interactions among CPs based on a non-cooperative game in terms of pricing, credibility of content and number of sponsored content. Additionally, the existence and uniqueness of the Nash equilibrium are proved through a detailed analysis. Based on the game properties, we propose a learning algorithm based on the best response dynamic that may lead the CPs to learn their strategies in a distributed manner. Numerical investigation shows the convergence of a proposed algorithm to the Nash equilibrium point and corroborates the fact that sponsoring content may improve the CPs outcome.

Keywords - Pricing, Credibility of content, Nash equilibrium, Sponsoring, Game theory

\section{INTRODUCTION}

The proliferation of social media, such as Facebook, Netflix, and Youtube, has increased the demand for content traffic, CPs are economically dependent on the amount of traffic consumed by end-users: more volume of traffic consumed conducted to more advertisement and then revenue. Therefore, one of the important challenges for CPs is how to incentive end-users for more access to the contents, and thus achieve a higher demand. For this reason, CPs are turning to new types of content pricing "sponsored content", sponsored content allows a CP to pay an ISP access fees, and thereby the endusers can access contents from the CP through the ISP for free. In 2014, AT\&T launched a data sponsored plan [1], where the CP (e.g., Youtube, Twitter) can pay the access price of end-users to their content through ISP. Examples in the USA are Netflix or Binge-On with T-Mobile, DIRECTV, etc.

Between the CPs and end-users, the ISP provides network access. In a traditional model, the ISP charges the end-users for content access. With the sponsored content, the CPs cooperates with the ISP, and the CP will pay the networks access price. Therefore, the sponsored content plan causes a positive cycle for the ISPs, the CPs, and end-users, i.e., a win-win situation. The CP sponsored content can motivate more end-users to have a higher demand for their content. Accordingly, the higher demand gives more payoff of

Received: August 28, 2019

Reviewed: October 17, 2019

Accepted: October 21, 2019 
the CP. For this reason, the academia and the industry has a remarkable interest to investigate the sponsored content scheme.

In this paper, we investigate the complex interactions among the CPs under a sponsored content schema. More specifically, our contributions are summarized as follows:

- We present new models that include one important feature, the CP's revenue models with a sponsored content plan.

- We formulate the interactions among CPs as a non-cooperative game in terms of price, the credibility of content and number of sponsored content.

- We formally prove the existence and the uniqueness of the Nash equilibrium of the non-cooperative game among CPs, which means that there exists a stable state where all $\mathrm{CP}$ do not have an incentive to change their strategies. So, our model ensures the existence of an equilibrium for keeping the economy stable and achieving economic growth. We propose an iterative algorithm to achieve the Nash equilibrium.

- We conduct thorough numeric investigation to determine the effect of sponsored content on the strategies of CPs. In addition, the convergence of the proposed algorithm to the Nash equilibrium point is explored. We believe, based on results that the proposed economic model is general and feasible, and this is applicable to modeling the competition among CPs content market with a sponsored content plan.

The rest of this paper is organized as follows. Section 2 discusses related work. In Section 3, we describe the system model. We prove the existence and uniqueness of a Nash equilibrium point in Section 4. Then, we present a numerical investigation in Section 5. In Section 6 conclusions and future.

\section{RELATED WORK}

Game theory has been applied to a wide range of networking problems to study the interaction of players seeking to maximize their profit [2-4]. The authors [5] study models that involve CPs, CDN providers and end-users. The authors formulate interactions among CPs and and among CDN as a non-cooperative game. In [6-7] the authors study competition between the CPs as a non-cooperative game. The authors in [8-9] studied competition between ISP in Information Centric Network using mathematical tools game theory. In the authors [10-11] modeled and studied competition between ISP where endusers are bounded rational.

The authors in [12] analyze a model with one CP several Internet Service Providers, where the $\mathrm{CP}$ can choose to sponsor a proportion of content and a level of advertisement. They show that sponsored content can be beneficial to the end-users and ISPs. In [13] the authors consider a sponsored content market with a single ISP, a single CP, and a set of end-users. The interactions among three entities are modeled as two-stage Stackelberg game. The authors have analyzed two scenarios: a scenario where the ISP and CP are in completion, and the scenario where the ISP and CP cooperate for a common goal. The authors [14] [15] in investigate joint sponsored and caching content under the noncooperative game. The interactions among ISP, CP, and end-users are modeled as a threestage Stackelberg game. In [16] the authors model the service selection process between end-users as an evolutionary population game, and pricing-then-sponsoring process between the CP and the ISP as a non-cooperative game. The authors show that sponsoring can help improve the profit of both provider (CP and ISP) and the end-user quality of experience. 


\section{PROBLEM FORMULATION}

We consider an Internet market model with multiple CP and an arbitrary number of end-users. End-users can access the contents of the CP only through the ISP network while $\mathrm{CP}$ provides the content to the end-users. The Internet market depends on three effective factors sponsoring, pricing and credibility of content. Under the assumption that each CP can sponsored content, it can decide to either sponsored the entire or a portion of the requested content. Let $N$, the number of content that the $\mathrm{CP}$ sells. The sponsoring strategy adopted by each ISP is denoted by $S$ that take values in the interval $[0, N]$. The $\mathrm{CP}$ pricing strategies set also consists of the content price $p_{c}$ that the end-users should pay for content. Each $\mathrm{CP}$ advertises to the end-users credibility of content $c$.

\subsection{DEMAND MODEL}

We consider that demand of content from end-users to $\mathrm{CP}$ is a linear function affected by strategies of all $\mathrm{CP}$, as for example in ([9] [8][22])as follows:

$$
D_{f}=d_{f}-\sigma_{f}^{f} p_{c_{f}}+\varsigma_{f}^{f} c_{f}+\chi_{f}^{f} S_{f}+\sum_{g=1, f \neq g}^{F}\left(\sigma_{f}^{g} p_{c_{g}}-\varsigma_{f}^{g} c_{g}-\chi_{f}^{g} S_{g}\right)
$$

The parameter $d_{f}$ expresses the potential demand of end-users. $\sigma_{f}^{g}, \varsigma_{f}^{g}$ and $\chi_{f}^{g}$ they are tree positive parameters representing respectively the responsiveness of $C P_{f}$ to price $p_{g}$, credibility of content $c_{g}$ and number of sponsored content $S_{g}$. For $C P_{f}$, the demand $D_{f}$ decreasing in the price it charges, $p_{c_{f}}$, and increase in the price charged by its opponent, $p_{c_{g}}$. The analogous relationship holds in credibility of content and number of sponsored content, in this case $D_{f}$ is increasing in $c_{f}$ (resp. $S_{f}$ ) and decreasing in $c_{g}\left(\right.$ resp. $\left.S_{g}\right)$.

\section{Assumption 1}

The sensitivity $\sigma$ verifies:

$$
\sigma_{f}^{f} \geq \sigma_{f}^{g}, \quad f \neq g
$$

The sensitivity $\varsigma$ verifies:

$$
\varsigma_{f}^{f} \geq \varsigma_{f}^{g}, \quad f \neq g
$$

The sensitivity $\chi$ verifies:

$$
\chi_{f}^{f} \geq \chi_{f}^{g}, \quad f \neq g .
$$

Assumption 1 implies that the influence of $\mathrm{CP}$ strategies on its observed demand is greater than the influence of the strategies of its opponent on their demand.

\subsection{UTILITY FUNCTION}

The utility of $C P_{f}$ can be modeled as:

$$
U_{f}=p_{c_{f}} D_{f}-p_{u} S_{f} D_{f}-p_{t} c_{f} D_{f}-\theta_{f} c_{f}
$$


$\theta_{f}$ the cost to produce a unit of credibility of content $c_{f} \cdot p_{t}$ represents the transmission fee that the CPs pay per unit of credibility of content $c_{f} \cdot p_{u}$ is sponsoring cost of CP per unit data and paid to the ISP. In the utility function, the first term is the revenue of $C P_{f}$, the second term denotes the cost due to sponsorship, the third term is the cost to produce the credibility of content $c_{f}$, and the fourth term is transmission fee results when the $C P_{f}$ forwards to the end-users demand with credibility of content $c_{f}$.

Credibility of content $c_{i}$ of $C P_{f}$ is a function of the quality of content $q_{c_{f}}$ and the quality service $q_{s_{f}}$, which is written as follows [7] [17]:

$c_{f}=\lambda q_{s_{f}}+\mu q_{c_{f}}$

where $\mu$ and $\lambda$ are two positive constants. The quality of content QoC for example, quality of video streaming [18]. $q_{s_{f}}$ is quality of service QoS measure (e.g. delay, see [19] [20]).

Then, the utility of $C P_{f}$ is given by the following formula:

$U_{f}=p_{c_{f}} D_{f}-p_{u} S_{f} D_{f}-p_{t}\left(\lambda q_{s_{f}}+\mu q_{c_{f}}\right) D_{f}-\theta_{f}\left(\lambda q_{s_{f}}+\mu q_{c_{f}}\right)$

\section{GAME ANALYSIS}

Let $G=\left[\Phi,\left\{\Pi_{c_{f}}, \Sigma_{f}, \Theta_{s_{f}}, \Theta_{c_{f}}\right\}\left\{\left\{U_{f}().\right\}\right]\right.$ denote the non-cooperative price QoC sponsoring QoS game (NPQSQG), where $\Phi=\{1, \ldots, F\}$ is the index set identifying the CPs, $\Pi_{c_{f}}$ is the price strategy set of $C P_{f}, \Theta_{s_{f}}$ is the QoS strategy set of $C P_{f}, \Sigma_{f}$ is the sponsoring strategy set of $C P_{f}, \Theta_{c_{f}}$ is the QoC strategy set of $C P_{f}$ and $U_{f}($.$) is$ the utility function of $C P_{f}$ defined in equation (4). We assume that the strategy spaces $\Pi_{c_{f}}, \Theta_{s_{f}}, \Sigma_{f}$ and $\Theta_{c_{f}}$ of each $C P_{f}$ are compact and convex sets with maximum and minimum constraints. Thus, for each $C P_{f}$ we consider as respective strategy spaces the closed intervals: $\Pi_{c_{f}}=\left[\underline{p}_{c_{f}}, \bar{p}_{c_{f}}\right], \quad \Theta_{s_{f}}=\left[\underline{q}_{s_{f}}, \bar{q}_{s_{f}}\right], \quad \Sigma_{f}=\left[\underline{S}_{f}, \bar{S}_{f}\right] \quad$ and $\Theta_{c_{f}}=\left[\underline{q}_{c_{f}}, \bar{q}_{c_{f}}\right] \quad$ Let the price vector $p_{c}=\left(p_{c_{1}}, \ldots, p_{c_{f}}\right)^{T} \in \Pi_{c}^{F}=\Pi_{c_{1}} \times \Pi_{c_{2}} \times \ldots \times \Pi_{c_{F}} \quad$, $\quad$ QoS $\quad$ vector $q_{s}=\left(q_{s_{1}}, \ldots, q_{s_{F}}\right)^{T} \in Q_{s}^{F}=\Theta_{s_{1}} \times \Theta_{s_{2}} \times \ldots \times \Theta_{s_{F}} \quad, \quad$ sponsoring $\quad$ vector $S=\left(S_{1}, \ldots, S_{F}\right)^{T} \in{ }^{F}={ }_{1} \times{ }_{2} \times \ldots \times{ }_{F} \quad$ QoC $\quad$ vector $q_{c}=\left(q_{c_{1}}, \ldots, q_{c_{F}}\right)^{T} \in Q_{c}^{F}=\Theta_{c_{1}} \times \Theta_{c_{2}} \times \ldots \times \Theta_{c_{F}}$. 


\subsection{PRICE GAME}

A NPQSQG in price $p_{c}$ is defined for fixed $\mathbf{q}_{s} \in \Theta_{s}, \mathbf{S} \in \Sigma, \mathbf{q}_{c} \in \Theta_{c}$ as $G\left(\mathbf{q}_{s}, \mathbf{S}, \mathbf{q}_{c}\right)=\left[\Phi,\left\{\Pi_{c_{i}}\right\},\left\{U_{f}\left(., \mathbf{q}_{s}, \mathbf{S}, \mathbf{q}_{c}\right)\right\}\right]$.

Definition 1 A price vector $\mathbf{p}_{c}^{*}=\left(p_{c_{1}}^{*}, \ldots, p_{c_{F}}^{*}\right)$ is a Nash equilibrium of the NPQSQG $G\left(\mathbf{q}_{s}, \mathbf{S}, \mathbf{q}_{c}\right)$ if:

$$
\begin{aligned}
& \forall\left(f, p_{c_{f}}\right) \in\left(\Phi, \Pi_{c_{f}}\right), \\
& U_{f}\left(p_{c_{f}}^{*}, \mathbf{p}_{f_{-i}}^{*}, \mathbf{q}_{s}, \mathbf{S}, \mathbf{q}_{c}\right) \geq U_{f}\left(p_{c_{f}}, \mathbf{p}_{c_{-f}}^{*}, \mathbf{q}_{s}, \mathbf{S}, \mathbf{q}_{c}\right)
\end{aligned}
$$

Theorem 1 For each $\mathbf{q}_{s} \in \Theta_{s}, \mathbf{S} \in \Sigma, \mathbf{q}_{c} \in \Theta_{c}$, the game $[\Phi$, $\left.\left\{\Pi_{c_{f}}\right\},\left\{U_{f}\left(., \mathbf{q}_{s s}, \mathbf{S}, \mathbf{q}_{c}\right)\right\}\right]$ admit a unique Nash Equilibrium.

\section{Proof}

To prove existence, we note that the strategy space $\Pi_{c_{f}}$ is defined in the closed interval bounded by the minimum and maximum prices. Thus, the strategy space $\Pi_{c_{f}}$ is nonempty, convex, and compact subset of the Euclidean space $R^{N}$. In addition, the second order derivative of the utility with respect to the prices is negative as below :

$$
\frac{\partial^{2} U_{f}}{\partial p_{c_{f}}^{2}}=-2 \sigma_{f}^{f} \leq 0
$$

The second derivative of the utility function is negative, then the utility function is thus concave, which ensures existence of a Nash equilibrium point in the game $G\left(\mathbf{q}_{s}, \mathbf{S}, \mathbf{q}_{c}\right)$.

We use the following proposition that holds for a concave game [21]: If a concave game satisfies the dominance solvability condition:

$$
-\frac{\partial^{2} U_{f}}{\partial p_{c_{f}}^{2}} \geq \sum_{g, g \neq f}\left|\frac{\partial^{2} U_{f}}{\partial p_{c_{f}} \partial p_{c_{g}}}\right|
$$

then the game $G\left(\mathbf{q}_{s}, \mathbf{S}, \mathbf{q}_{c}\right)$ admits a unique Nash equilibrium point.

The mixed partial is written as:

$$
\frac{\partial^{2} U_{f}}{\partial p_{c_{f}} \partial p_{c_{g}}}=\sigma_{f}^{g} \geq 0
$$

Then,

$$
-\frac{\partial^{2} U_{f}}{\partial p_{c_{f}}^{2}}-\sum_{g, g \neq f}\left|\frac{\partial^{2} U_{f}}{\partial p_{c_{f}} \partial p_{c_{g}}}\right|=2 \sigma_{f}^{f}-\sum_{g, g \neq f} \sigma_{f}^{g} \geq 0
$$

Thus, the game $G\left(\mathbf{q}_{s}, \mathbf{S}, \mathbf{q}_{c}\right)$ admit a unique Nash equilibrium point. 


\subsection{NUMBER OF SPONSORED CONTENT GAME}

A NPQSQG in number of sponsored content is defined for a fixed $\mathbf{p}_{c} \in \Pi_{c}, \mathbf{q}_{s} \in \Theta_{s}$, $\mathbf{q}_{c} \in \Theta_{c}$ as $G\left(\mathbf{p}_{c}, \mathbf{q}_{s}, \mathbf{q}_{c}\right)=\left[\Phi,\left\{\Sigma_{f}\right\},\left\{U_{f}\left(\mathbf{p}_{c}, \mathbf{q}_{s}, ., \mathbf{q}_{c}\right)\right\}\right]$.

Definition 2 A sponsoring vector $\mathbf{S}^{*}=\left(S_{1}^{*}, \ldots, S_{F}^{*}\right)$ is a Nash equilibrium of the $N P Q S Q G G\left(\mathrm{p}_{c}, \mathrm{q}_{s}, \mathrm{q}_{c}\right)$ if:

$$
\begin{aligned}
& \forall\left(f, S_{f}\right) \in\left(\Phi, \Sigma_{f}\right), \\
& U_{f}\left(\mathbf{p}_{c}, \mathbf{q}_{s}, S_{f}^{*}, \mathbf{S}_{-f}^{*}, \mathbf{q}_{c}\right) \geq U_{f}\left(\mathbf{p}_{c}, \mathbf{q}_{s}, S_{f}, \mathbf{S}_{-f}^{*}, \mathbf{q}_{c}\right)
\end{aligned}
$$

Theorem 2 For each $\mathbf{p}_{c} \in \Pi_{c}, \mathbf{q}_{s} \in \Theta_{s}, \mathbf{q}_{c} \in \Theta_{c}$, the game $[\Phi$, $\left.\left\{\Sigma_{f}\right\},\left\{U_{f}\left(\mathbf{p}_{c}, \mathbf{q}_{s}, ., \mathbf{q}_{c}\right)\right\}\right]$ admit a unique Nash Equilibrium.

\section{Proof}

To prove existence, we note that the strategy space $\Sigma_{f}$ is defined in the closed interval bounded by the minimum and maximum number of sponsored content. Thus, the strategy space $\Sigma_{f}$ is nonempty, convex, and compact subset of the Euclidean space $R^{N}$. In addition, the second order derivative of the utility with respect to the number of sponsored content is negative as below :

$$
\frac{\partial^{2} U_{f}}{\partial S_{f}^{2}}=-2 \chi_{f}^{f} p_{u} \leq 0
$$

The second derivative of the utility function is negative, then the utility function is thus concave, which ensures existence of a Nash equilibrium point in the game $G\left(\mathbf{p}_{c}, \mathbf{q}_{s}, \mathbf{q}_{c}\right)$.

We use the following proposition that holds for a concave game [21]: If a concave game satisfies the dominance solvability condition :

$$
-\frac{\partial^{2} U_{f}}{\partial S_{f}^{2}} \geq \sum_{g, g \neq f}\left|\frac{\partial^{2} U_{f}}{\partial S_{f} \partial S_{g}}\right|
$$

then the game $G\left(\mathbf{p}_{c}, \mathbf{q}_{s}, \mathbf{q}_{c}\right)$ admits a unique Nash equilibrium point.

The mixed partial is written as:

$$
\frac{\partial^{2} U_{f}}{\partial S_{f} \partial S_{g}}=\chi_{f}^{g} p_{u} \geq 0
$$

Then,

$$
-\frac{\partial^{2} U_{f}}{\partial S_{f}^{2}}-\sum_{g, g \neq f}\left|\frac{\partial^{2} U_{f}}{\partial S_{f} \partial S_{g}}\right|=\left(2 \chi_{f}^{f}-\sum_{g, g \neq f} \chi_{f}^{g}\right) p_{u} \geq 0
$$

Thus, the game $G\left(\mathbf{p}_{c}, \mathbf{q}_{s}, \mathbf{q}_{c}\right)$ admit a unique Nash equilibrium point. 


\subsection{QoC GAME}

A NPQSQG in QoC is defined for a fixed $\mathbf{p}_{c} \in \Pi_{c}, \mathbf{q}_{s} \in \Theta_{s}, \mathbf{S} \in \Sigma$ as $G\left(\mathbf{p}_{c}, \mathbf{q}_{s}, \mathbf{S}\right)=\left[\Phi,\left\{\Theta_{c_{f}}\right\},\left\{U_{f}\left(\mathbf{p}_{c}, \mathbf{q}_{s}, \mathbf{S},.\right)\right\}\right]$.

Definition 3 A QoC vector $\mathbf{q}_{c}^{*}=\left(q_{c_{1}}^{*}, \ldots, q_{c_{F}}^{*}\right)$ is a Nash equilibrium of the NPQSQG $G\left(\mathbf{p}_{c}, \mathbf{q}_{s}, \mathbf{S}\right)$ if:

$$
\begin{aligned}
& \forall\left(f, q_{c_{f}}\right) \in\left(\Phi, \Theta_{c_{f}}\right), \\
& U_{f}\left(\mathbf{p}_{c}, \mathbf{q}_{s}, \mathbf{S}, q_{c_{f}}^{*}, \mathbf{q}_{c_{-f}}^{*}\right) \geq U_{f}\left(\mathbf{p}_{c}, \mathbf{q}_{s}, \mathbf{S}, q_{c_{f}}, \mathbf{q}_{c_{-f}}^{*}\right)
\end{aligned}
$$

Theorem 3 For each $\mathbf{p}_{c} \in \Pi_{c}, \mathbf{q}_{s} \in Q_{s}, \mathbf{S} \in \Sigma$, the game $[\Phi$, $\left.\left\{\Theta_{c_{f}}\right\},\left\{U_{f}\left(\mathbf{p}_{c}, \mathbf{q}_{s}, \mathbf{S},.\right)\right\}\right]$ admit a unique Nash Equilibrium.

\section{Proof}

To prove existence, we note that the strategy space $\Theta_{c_{f}}$ is defined in the closed interval bounded by the minimum and maximum QoCs. Thus, the joint strategy space $\Theta_{c_{f}}$ is nonempty, convex, and compact subset of the Euclidean space $R^{N}$. In addition, the second order derivative of the utility with respect to the QoC is negative as below :

$$
\frac{\partial^{2} U_{f}}{\partial q_{c_{f}}^{2}}=-2 \mu \chi_{f}^{f} p_{t} \leq 0
$$

The second derivative of the utility function is negative, then the utility function is thus concave, which ensures existence of a Nash equilibrium point in the game $G\left(\mathbf{p}_{c}, \mathbf{q}_{s}, \mathbf{S}\right)$.

We use the following proposition that holds for a concave game [21]: If a concave game satisfies the dominance solvability condition :

$$
-\frac{\partial^{2} U_{f}}{\partial q_{c_{f}}^{2}} \geq \sum_{g, g \neq f}\left|\frac{\partial^{2} U_{f}}{\partial q_{c_{f}} \partial q_{c_{g}}}\right|
$$

then the game $G\left(\mathbf{p}_{c}, \mathbf{q}_{s}, \mathbf{S}\right)$ admits a unique Nash equilibrium point.

The mixed partial is written as:

$\frac{\partial^{2} U_{f}}{\partial q_{c_{f}} \partial q_{c_{g}}}=\mu \chi_{f}^{j} p_{t}$

Then,

$-\frac{\partial^{2} U_{f}}{\partial q_{c_{f}}^{2}}-\sum_{g, g \neq f}\left|\frac{\partial^{2} U_{f}}{\partial q_{c_{f}} \partial q_{c_{g}}}\right|=\mu\left(\chi_{f}^{f}-\sum_{g, g \neq f} \chi_{f}^{g}\right) p_{t}$

Thus, the game $G\left(\mathbf{p}_{c}, \mathbf{q}_{s}, \mathbf{S}\right)$ admit a unique Nash equilibrium point. 


\subsection{QoS GAME}

A NPQSQG in QoS is defined for a fixed $\mathbf{p}_{c} \in \Pi_{c}, \mathbf{S} \in \Sigma, \mathbf{q}_{c} \in \Theta_{c}$ as $G\left(\mathbf{p}_{c}, \mathbf{q}_{c}, \mathbf{S}\right)=\left[\Phi,\left\{\Theta_{s_{f}}\right\},\left\{U_{f}\left(\mathbf{p}_{c}, ., \mathbf{S}, \mathbf{q}_{c}\right)\right\}\right]$.

Definition 4. A $Q o S$ vector $\mathbf{q}_{s}^{*}=\left(q_{s_{1}}^{*}, \ldots, q_{s_{F}}^{*}\right)$ is a Nash equilibrium of the NPQSQG $G\left(\mathbf{p}_{c}, \mathbf{q}_{c}, \mathbf{S}\right)$ if:

$$
\begin{aligned}
& \forall\left(f, q_{s_{f}}\right) \in\left(\Phi, \Theta_{s_{f}}\right), \\
& U_{f}\left(\mathbf{p}_{c}, q_{s_{f}}^{*}, \mathbf{q}_{s_{-f}}^{*}, \mathbf{S}, \mathbf{q}_{c}\right) \geq U_{f}\left(\mathbf{p}_{c}, q_{s_{f}}, \mathbf{q}_{s_{-f}}^{*}, \mathbf{S}, \mathbf{q}_{c}\right)
\end{aligned}
$$

Theorem 4 For each $\mathbf{p}_{c} \in \Pi_{c}, \mathbf{S} \in \Sigma, \mathbf{q}_{c} \in \Theta_{c}$, the game $[\Phi$, $\left.\left\{\Theta_{s_{f}}\right\},\left\{U_{f}\left(\mathbf{p}_{c}, ., \mathbf{S}, \mathbf{q}_{c}\right)\right\}\right]$ admit a unique Nash Equilibrium.

The QoS game has the same analysis as QoC game, just we replace $\mu$ by $\lambda$.

\subsection{LEARNING NASH EQUILIBRIUM}

The section mentioned above shows clearly that the Nash equilibrium point is unique. Now, we devise an algorithm that converges to the Nash equilibrium in a distributed manner based on the best-response dynamic. A best response dynamics scheme contains a series of rounds, wherein each round, each $\mathrm{CP}$ observe the policy taken by its competitors in previous rounds and input them in its decision process to update its policy. In the first round, the choice of each $\mathrm{CP}$ is the best response based on its arbitrary belief about what the other players will choose.

Algorithm 1 summarizes the best response learning steps that each $\mathrm{CP}$ has to perform in order to find the Nash equilibrium point.

Algorithm1 : Best Response Algorithm Dynamics

Initialize vectors $\mathrm{x}(0)=\left[x_{1}(0), \ldots, x_{F}(0)\right]$ randomly;

For each $C P_{f}, f \in K$ at time instant $t$ compute:

$$
\text { - } x_{f}(t+1)=\underset{x_{f} \in X_{f}}{\operatorname{argmax}}\left(U_{f}(\mathbf{x}(t))\right) \text {. }
$$

If $\forall f \in,\left|x_{f}(t+1)-x_{f}(t)\right|<\varepsilon$, then STOP. Else, $t=t+1$ and go to step (2)

Such as:

- $x$ refers to the vector price $p_{c}$, vector of sponsored content $S$, vector $q_{s}$ and vector $q_{c}$.

- $X_{k}$ refers to the policy profile price, number of sponsored content, QoS or QoC. 


\section{NUMERICAL INVESTIGATION}

In this section, we perform the simulations to evaluate the strategy of CPs under competitive cases, where each CP sponsored a number of content. We consider a scenario with two homogeneous CP seeking to maximize their payoff. The parameter values used in the numerical investigation are summarized in Table I.

Table I. Setting used for numerical simulations

\begin{tabular}{|c|c|c|c|c|}
\hline$\sigma_{1}^{1}=\sigma_{2}^{2}$ & $\sigma_{f}^{g}, f \neq g$ & $\varsigma_{1}^{1}=\varsigma_{2}^{2}$ & $\varsigma_{f}^{g}, f \neq g$ & $F$ \\
\hline 7 & 0.3 & 0.7 & 0.3 & 2 \\
\hline$\lambda$ & $\mu$ & $p_{u}$ & $\chi_{1}^{1}=\chi_{2}^{2}$ & $\chi_{f}^{g}, f \neq g$ \\
\hline 5 & 0.5 & 10 & 0.7 & 0.3 \\
\hline$d_{2}$ & $d_{1}$ & $p_{t}$ & $\theta_{1}=\theta_{2}$ & $N$ \\
\hline 250 & 250 & 10 & 0.5 & 100 \\
\hline
\end{tabular}

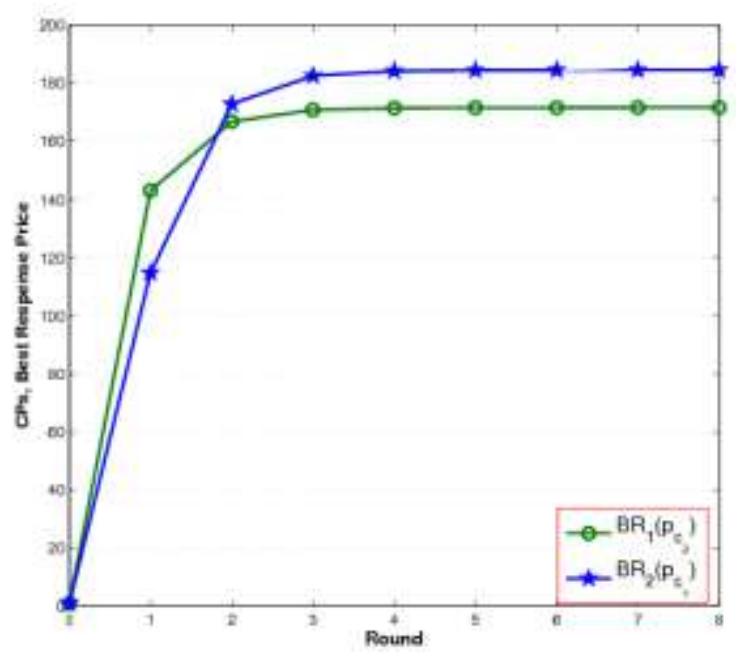

Fig. 1 Price game: Convergence to the Price at Nash equilibrium

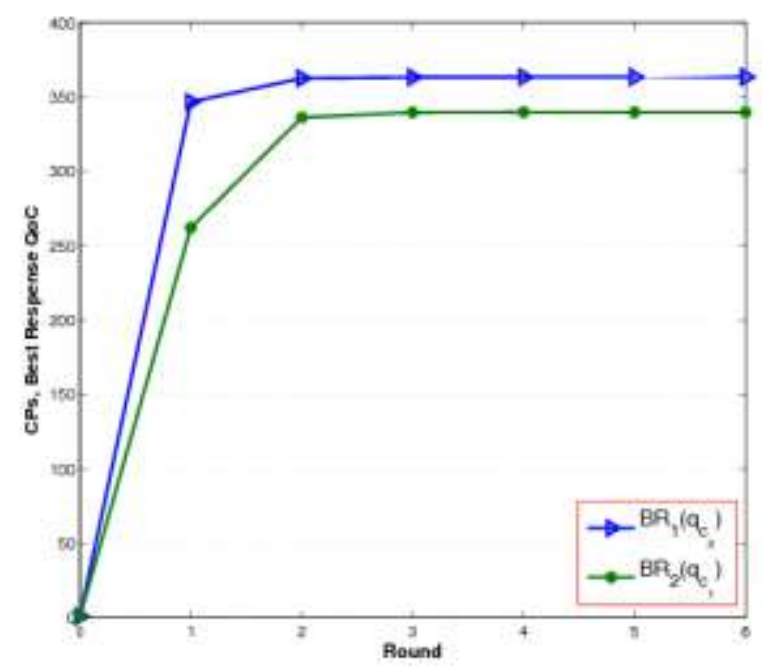

Fig. 2 Price game: Convergence to the QoC at Nash equilibrium 


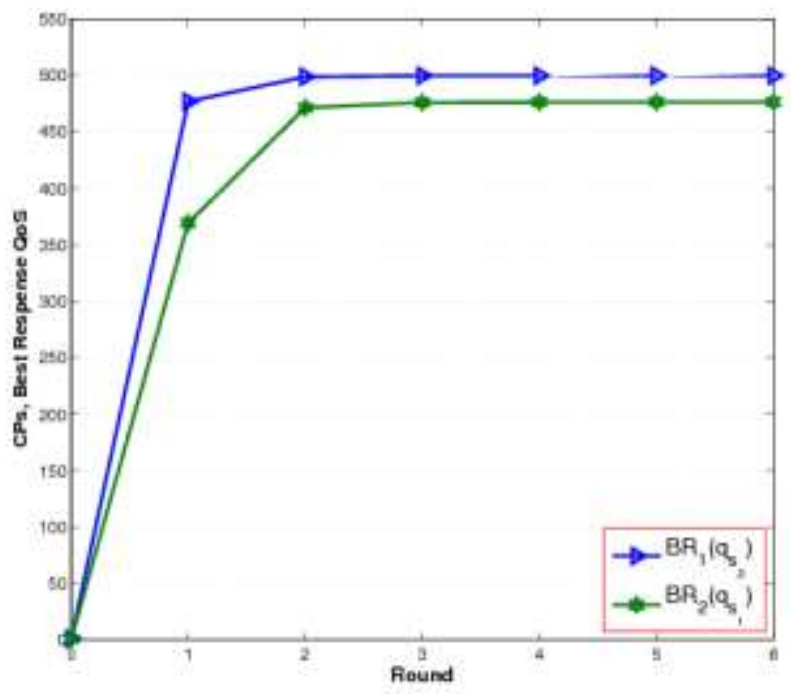

Fig. 3 Price game: Convergence to the QoS at Nash equilibrium.

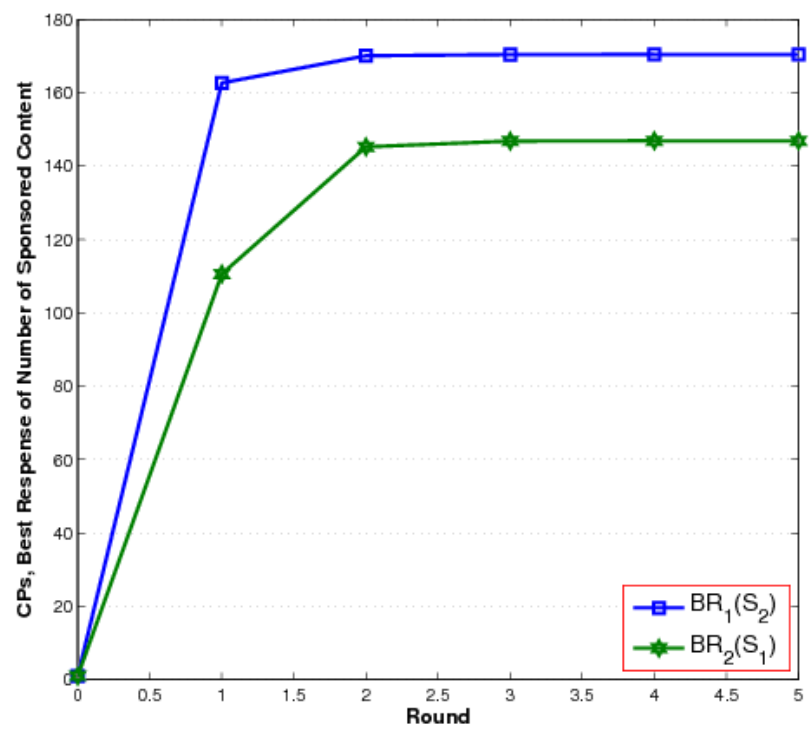

Fig. 4 Price game: Convergence to the number of sponsored content at Nash equilibrium.

Figures 1, 2, 3 and 4 illustrate the convergence to equilibrium price, number of sponsored content, QoS and QoC. This figure shows the number of iterations needed for convergence to the Nash equilibrium point, it is clear that the speed of convergence is relatively high. The Figures 1, 2, 3 and 4 demonstrates the existence and uniqueness of a Nash equilibrium point at which no CPs can profitably deviate given the strategies of another CP. 


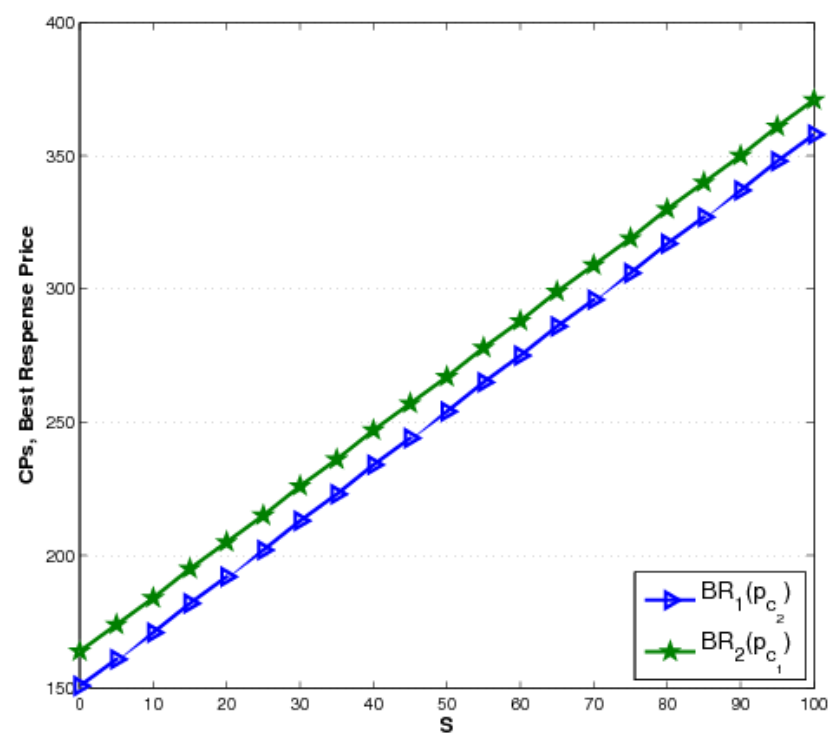

Fig. 5 Equilibrium price $p_{c}$ as a function of number of sponsored content $S$

We investigate the impact of the number of sponsored content $S$ on the price $p_{c}$, as illustrated in Fig. 5. When the number of sponsored content $S$ increase the price $p_{c}$ increase. The intuition is that as the number of sponsored content $S$ increase, the sponsoring cost increase. Therefore, the $\mathrm{CP}$ need to increase their price $p_{c}$ in order to compensate the increase in the sponsoring cost. The CP needs to choose a lower price to attract more end-users to consume more sponsored content.

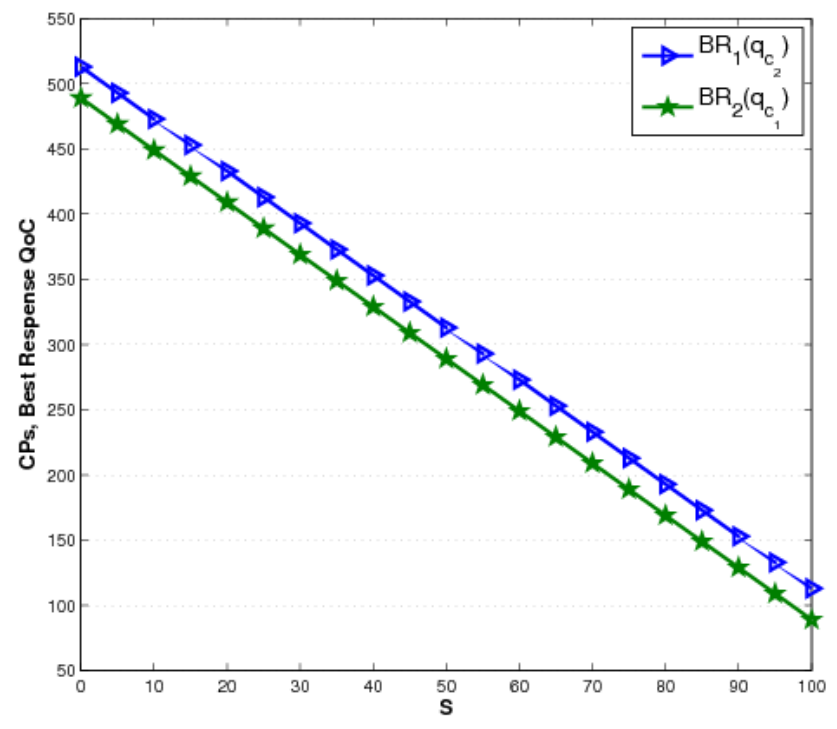

Fig. 6 Equilibrium QoC $q_{c}$ as a function of number of sponsored content $S$ 


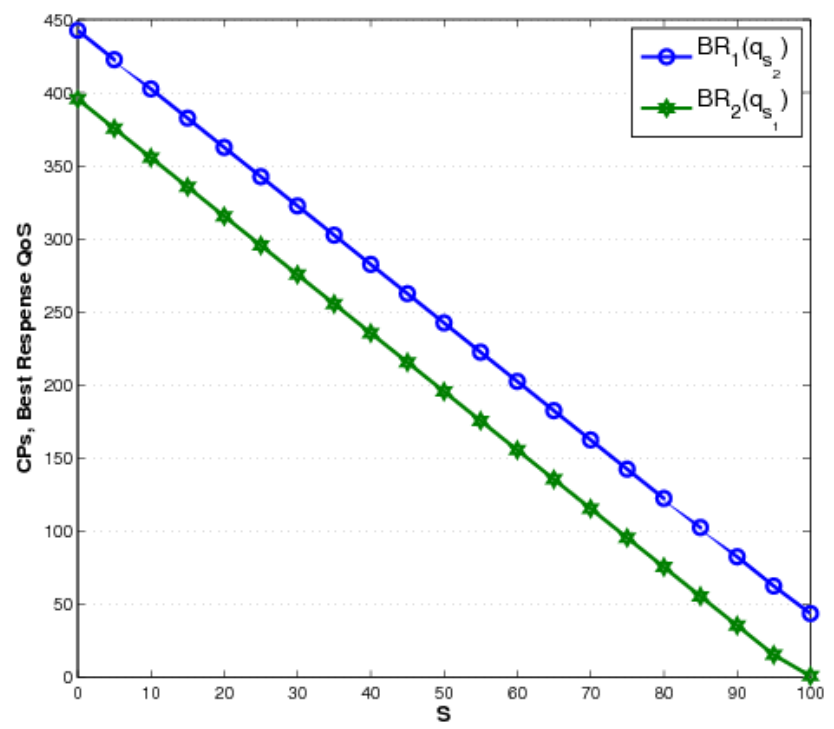

Fig. 7 Equilibrium QoS $q_{s}$ as a function of number of sponsored content $S$

Figs. 6 and 7 shows the QoS and QoC as function of number of sponsored content $S$. The QoS and the QoC decrease as the number of sponsored content get increases $S$. The reason is that as the number of sponsored content $S$ increases, the sponsored content demand of the end-users increases. Therefore, the transmission fee increases. Thus, the $\mathrm{CP}$ needs to slightly decrease its QoS and QoC to compensate the increase in the transit cost.

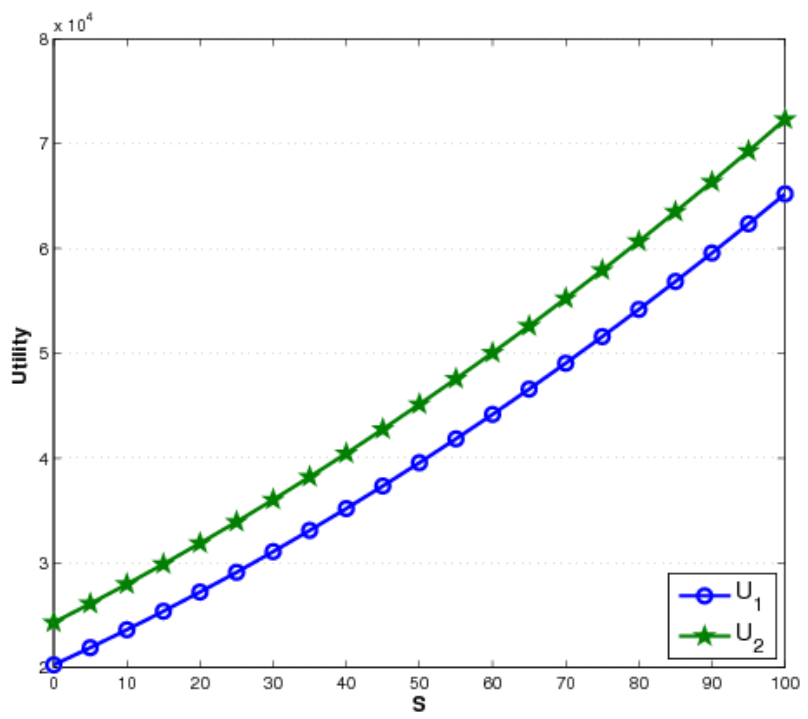

Fig. 8 Utility as a function of number of sponsored content $S$

We plot the expected utility of CPs as a function of the number of sponsored content $S$ in Fig. 8. Utility increase with respect to the number of sponsored content $S$. As $S$ increases, the sponsored content demand of the end-users increases. Moreover, the 
increase of the sponsored content demand leads to the profit improvement of both $\mathrm{CP}$ since they can sell more content to the incoming end-users.

\section{CONCLUSION}

In this paper, we developed an analytical framework for sponsoring content in the internet market that comprises of multiple content provider and end-users. We have formulated the interactions among the CPs as a non-cooperative game. Then, we have analytically analyzed the game. Furthermore, we proved the existence and uniqueness of the Nash equilibrium point in a competitive market under our proposed model. This result is significant because it implies that a stable solution with suitable economic incentives in sponsoring content is feasible in the internet paradigm. Additionally, we have proposed a distributed algorithm based on best response dynamic, which is ensured to converge to the Nash equilibrium point. Through the extensive simulation analysis, it has been verified that sponsoring content can improve the profit of $\mathrm{CP}$.

\section{REFERENCES}

[1] “At\&t sponsored plan," https://www.att.com/.

[2] Ait Omar, Driss, et al. "Joint Price and QoS Competition with Bounded Rational Customers," in Networked Systems: 5th International Conference, NETYS 2017, Marrakech, Morocco, May 17-19, 2017, Proceedings, A. El Abbadi and B. Garbinato, Eds. Cham: Springer International Publishing, 2017, pp. 457-471.

[3] El Amrani, Mohamed, et al. "Cournot Game of ISP under Net Neutrality," International Journal of Control and Automation, vol. 11, no. 7, p. 16, 2018.

[4] El Amrani, Mohamed, et al. "A Game Theoretic Approach to Optimize Transmission Strategies for Mobile Applications," in 2018 6th International Conference on Wireless Networks and Mobile Communications (WINCOM), Marrakesh, Morocco, 2018, pp. 1-6.

[5] Ait Omar, Driss, et al. "Analysis of Bargaining Game Policy in the Internet Content Distribution Chain:", International Journal of Mobile Computing and Multimedia Communications, vol. 10, no. 3 , pp. 47-73, Jul. 2019.

[6] M'hamed, Outanoute et al., "A Non-Cooperative Game Analysis of Competition between Content Providers in the Internet Market," p. 3.

[7] Garmani, Hamid, et al. "Analysis of Competition between CPs Fronting Advertisers," International Journal of Advanced Science and Technology, vol. 117, pp. 53-66, Aug. 2018.

[8] Garmani, Hamid, et al. "Caching Games between ISP in Information Centric Network," International Journal of Control and Automation, vol. 11, no. 4, pp. 125-142, Apr. 2018.

[9] Garmani, Hamid, et al. "New Competition-Based Approach for Caching Popular Content in ICN," in Networked Systems, vol. 11028, A. Podelski and F. Taïani, Eds. Cham: Springer International Publishing, 2019, pp. 286-300.

[10] Ait Omar, Driss, et al. "On understanding price-QoS war for competitive market and confused consumers," Computing, vol. 101, no. 9, pp. 1327-1348, Sep. 2019.

[11] Ait Omar, Driss, et al. "A Customer Confusion Environment in Telecommunication Networks: Analysis and Policy Impact,” Int. J. Coop. Info. Syst., vol. 28, no. 02, p. 1930002, Jun. 2019.

[12] Maillé, Patrick, et al. "Analysis of Sponsored Data Practices in the Case of Competing Wireless Service Providers," in Computational Intelligence and Intelligent Systems, vol. 986, H. Peng, C. Deng, Z. Wu, and Y. Liu, Eds. Singapore: Springer Singapore, 2019, pp. 55-70.

[13] Xiong, Zehui, et al. "Competition and cooperation analysis for data sponsored market: A network effects model," in 2018 IEEE Wireless Communications and Networking Conference (WCNC), Barcelona, 2018, pp. 1-6.

[14] Xiong, Zehui, et al. "Joint Sponsored and Edge Caching Content Service Market: A Game-Theoretic Approach,” IEEE Trans. Wireless Commun., vol. 18, no. 2, pp. 1166-1181, Feb. 2019.

[15] Xiong, Zehui, et al. "Game Theoretic Analysis for Joint Sponsored and Edge Caching Content Service Market," in 2018 IEEE Global Communications Conference (GLOBECOM), Abu Dhabi, United Arab Emirates, 2018, pp. 1-7.

[16] Wang, Wenbo, et al. "A Hierarchical Game With Strategy Evolution for Mobile Sponsored Content and Service Markets,” IEEE Trans. Commun., vol. 67, no. 1, pp. 472-488, Jan. 2019.

[17] Outanoute, M'hamed, et al. "A Non-Cooperative Game Analysis of Competition between Content Providers in the Internet Market," IJBDCN, vol. 15, no. 1, pp. 88-104, Jan. 2019.

[18] Ait Omar, Driss, et al. "A Game-Theoretic Approach for the Internet Content Distribution Chain," in Networked Systems, vol. 11028, A. Podelski and F. Taïani, Eds. Cham: Springer International Publishing, 2019, pp. 270-285. 
[19] Baslam, Mohamed, et al. "A Game Theoretic Analysis of Price-QoS Market Share in Presence of Adversarial Service Providers," in Game Theory Relaunched, H. Hanappi, Ed. InTech, 2013.

[20] Baslam, Mohamed, et al. "Market share game with adversarial access providers: A neutral and a nonneutral network analysis," in Network Games, Control and Optimization (NetGCooP), 2011 5th International Conference on, 2011, pp. 1-6.

[21] Lasaulcen, Samson, et al. "Methodologies for analyzing equilibria in wireless games," IEEE Signal Processing Magazine, vol. 26, no. 5, pp. 41-52, 2009.

[22] Garmani, Hamid, et al. "Analysis of a dynamics duopoly game with two content providers", Chaos, Solitons and Fractals, https://doi.org/10.1016/j.chaos.2019.109466 